\title{
The impact of perceived control on psychological distress and health behavior adherence in patients with cardiovascular disease
}

\author{
Kalon R. Eways ${ }^{* 1}$, Kymberley K. Bennett ${ }^{1}$, Kadie M. Harry ${ }^{1}$, Jillian M.R. Clark ${ }^{2,3}$, Elizabeth J. Wilson ${ }^{1}$ \\ ${ }^{1}$ Department of Psychology, University of Missouri-Kansas City, Kansas City, MO, United States \\ ${ }^{2}$ Center of Excellence for Stress and Mental health, VA San Diego Healthcare System, San Diego, CA, United States \\ ${ }^{3}$ Department of Psychiatry, University of California, San Diego, CA, United States
}

Received: August 1, 2018

DOI: $10.5430 /$ jnep.v9n2p76
Accepted: October 10, 2018

Online Published: October 15, 2018

\begin{abstract}
Background: Symptoms of depression and anxiety have been shown to negatively impact physical health outcomes among individuals with cardiovascular disease (CVD). Therefore, an important step in developing interventions to reduce risk for cardiac event recurrence is to identify the emotional and cognitive predictors of psychological distress. This study examined one possible cognitive predictor: perceived control (PC). Specifically, this study tested whether symptoms of depression and anxiety mediate the relationship between $\mathrm{PC}$ and adherence to health behavior recommendations in patients participating in a cardiac rehabilitation (CR) program.

Methods: Self-report measures were administered to $146 \mathrm{CR}$ patients at the beginning of CR and 12-weeks later, at the end of CR.

Results: Anxiety and depressive symptoms did not mediate the relationship between PC and health behavior adherence. Rather, PC was cross-sectionally related to symptoms of psychological distress, and it predicted health behavior adherence 12-weeks later.

Conclusions: Results imply that PC has long-term effects on health behavior adherence, an important outcome in CR that reduces risk for recurrence.
\end{abstract}

Key Words: Cardiovascular disease, Control, Rehabilitation, Distress, Health behaviors

\section{INTRODUCTION}

\subsection{Psychological distress and cardiovascular disease}

Cardiovascular disease (CVD) is the leading cause of death in the United States (US), and this is quickly becoming the trend globally. ${ }^{[1]}$ By 2030, it is projected that $40.5 \%$ of the US population will have some form of CVD. ${ }^{[2]}$ Similar to other chronic conditions, the etiology of CVD can be attributed to a myriad of factors. ${ }^{[1]}$ Although behavioral risk factors are strongly associated with onset, psychological distress is also associated with the development, maintenance, and trajectory of CVD. For example, severe and subclinical levels of depressive symptoms are known risk factors for the development of CVD in healthy adults. ${ }^{[3]}$ Further, patients with CVD who suffer from both anxiety and depressive symptoms experience more adverse physical health outcomes (e.g., increased risk for mortality) than patients not

\footnotetext{
*Correspondence: Kalon R. Eways; Email: kren7d@mail.umkc.edu; Address: Department of Psychology, 5030 Cherry Street, Room 214, University of Missouri, Kansas City, Kansas City, MO, United States.
} 
experiencing mental health symptoms. ${ }^{[3,4]}$ Unfortunately, psychological distress is also ubiquitous within this patient population: It is estimated that $15 \%-20 \%$ of patients hospitalized for a myocardial infarction (MI) meet criteria for a depressive disorder and that 20\%-50\% of patients with CVD experience at least moderate levels of anxiety. ${ }^{[5,6]}$

\subsection{Psychological distress and cardiac health behaviors}

Because of the strong association between psychological distress and long-term physical health outcomes in patients with CVD, it is important for clinicians and researchers to study the mechanisms linking these constructs. One possible mechanism is engagement in health-promoting behaviors, as diagnoses of depression have been consistently linked to low levels of physical activity, poor diet, high rates of cigarette smoking, and nonadherence to cardiac medications. ${ }^{[7]}$ Likewise, anxiety symptoms are associated with high rates of smoking and alcohol use, low levels of physical activity, and poor diet. ${ }^{[3,8]}$ Over time, these maladaptive health behaviors may lead to a myriad of harmful long-term health issues including CVD onset, and to recurrence in those who have been previously diagnosed with $\mathrm{CVD}^{\left[{ }^{[7,9]}\right.}$

\subsection{Perceived control and psychological distress}

In addition to understanding the proximal mechanisms linking distress to long-term physical health outcomes among patients with CVD, it is also important to identify psychosocial constructs that predict distress. One potential psychosocial predictor of distress is perceived control (PC), which is defined in this context as the extent to which an individual believes he/she has personal control over disease-related outcomes. ${ }^{[10]}$ Extensive research has connected PC with psychological processes, particularly in the development of anxiety and depression. ${ }^{[11-13]}$ In fact, Abramson and colleagues ${ }^{[11]}$ were among the first researchers to examine the effects of low PC in human populations. They proposed the reformulated learned helplessness model, which predicts that outcomes perceived to be uncontrollable result in motivational, cognitive, and emotional deficits. These deficits then predict the onset of feelings of helplessness, which in turn, contribute to the development of psychological distress. While Abramson et al. ${ }^{[11]}$ recognized that specific perceptions and causal attributions may differ across individuals and thus produce disparate outcomes, the temporal order of the relationship between control and distress is presumed to be constant. In other words, perceptions of control are hypothesized to precede the development of distress, with less controllability predicting poorer psychological outcomes. Their model has received extensive empirical support ${ }^{[14]}$ and is a widely-cited theory to explain the etiology of psychological distress. Whereas most of the research using the Published by Sciedu Press model has focused on depressive symptoms as the outcome, research has supported its predictions for anxiety symptoms as the outcome, as well. ${ }^{[15]}$

\subsection{Perceived control and cardiac health outcomes}

Guided by the reformulated model, researchers have documented positive effects of PC on mental and physical health outcomes within samples of patients with chronic illnesses and diseases, including CVD. ${ }^{[16]}$ For example, one study ${ }^{[10]}$ examined PC and psychosocial recovery 6 months following a cardiac event. Results showed participants high in PC reported fewer depressive symptoms, less hostility, and better psychosocial adjustment at follow-up compared to patients low in PC. These findings were confirmed by a later investigation $^{[17]}$ examining a sample of patients diagnosed with heart failure: those high in PC had better distances on 6-minute walk tests, and lower levels of anxiety symptoms, depressive symptoms, and hostility, than patients low in PC. Another study ${ }^{[18]}$ examined PC's effects in patients undergoing coronary artery bypass grafts (CABG) immediately before surgery, after surgery, and 2-weeks post-discharge. Results showed patients high in $\mathrm{PC}$ reported fewer symptoms of anxiety and depression at all three time points compared to their low PC counterparts. Other investigations have linked PC with better health-related quality of life and physical functioning among patients with CVD. ${ }^{[19-21]}$

\subsection{Perceived control and cardiac health behaviors}

Whereas connections between PC and distress are robust, as are connections between distress and poor distal physical health outcomes in patients with CVD, research examining associations between PC and cardiac health behavior adherence (a proximal outcome) is sparse and somewhat mixed. One recent investigation ${ }^{[20]}$ reported a significant relationship between health-related PC and both depressive symptoms and health-related quality of life in a sample of 265 patients preparing to undergo a $\mathrm{CABG}$ in a pre-surgery assessment clinic in the UK. However, PC was not directly related to medication adherence, cardiac rehabilitation (CR) attendance, or physical activity. It is, therefore, possible that PC affects proximal and distal cardiac health outcomes via another variable, such as psychological distress.

\subsection{Current study}

The purpose of the current study was to examine relationships between PC, symptoms of psychological distress, and adherence to health behavior recommendations among patients enrolled in a CR program. In particular, this study focused on two types of PC related to CVD: PC over current cardiac event recovery and $\mathrm{PC}$ over preventing cardiac event recurrence. We aimed to fill a gap in the literature 
by testing direct and indirect effects of PC on an important proximal physical health outcome among patients in CR: health behavior adherence. Because adherence to health behavior change recommendations in $\mathrm{CR}$ is associated with improved recovery and lower 5 -year mortality, ${ }^{[1]}$ better understanding the direct and indirect psychosocial predictors of adherence is important. Guided by the reformulated learned helplessness model ${ }^{[11]}$ and past research involving patients with CVD, ${ }^{[7,10,16]}$ it was hypothesized that baseline (Time 1) depressive and anxiety symptoms would mediate the relationship between PC at the beginning of CR (Time 1) and health behavior adherence 12-weeks later, at the end of CR (Time 2). Specifically, it was expected that PC would be negatively related to depressive and anxiety symptoms, which would in turn be negatively related to health behavior adherence.

\section{MethodS}

\subsection{Design}

These data were collected as part of a larger descriptive, longitudinal study of psychosocial outcomes among patients in two Phase II CR programs in two Midwestern cities. Data collection took place between 2005 and 2011. The study was approved by Institutional Review Boards at each study site.

\subsection{Data collection procedures}

Eligible patients were identified and recruited during their CR intake sessions by members of the CR staff. During the patients' intake interviews, they were asked by a staff member if they were interested in learning more about the study. If they expressed interest, their contact information was forwarded to the research team. Within one week, a member of the research team called patients and provided additional information about the study. Patients were also given the opportunity to ask questions at that time and were informed that their participation was voluntary and confidential. If the patients stated they would like to participate in the study, they were mailed a consent form, a Time 1 self-report questionnaire packet, and a postage-paid return envelope. Time 2 questionnaires were mailed 12 weeks later, at the end of CR.

\subsection{Participants}

Inclusion criteria for participation included enrolling in a Phase II CR program, 18 years of age or older, diagnosis of CVD, and no cognitive impairments impeding the ability to consent and complete the study procedures. In the larger (or parent) study, a total of 552 patients were approached for potential inclusion, with $321(58 \%)$ agreeing to receive additional information about the study. Of these, 211 participated and provided complete data for Time 1, with 153 participants providing complete data for the variables of interest at both
Times 1 and 2. However, seven of these participants were missing a significant amount of data (greater than 40\%), so those cases were excluded. The final sample for the current study consisted of 146 participants (69\% of the original sample, or $26 \%$ of those initially approached) with complete data at both time points. This sample size is consistent with empirical recommendations for mediation analyses aiming to achieve $80 \%$ power. $^{[22]}$

When comparing participants with data at both time points to those who only provided baseline data (and were therefore excluded), no differences were found for the following variables: sex, race, marital status, employment status, income, educational attainment, diagnosis, risk stratification for disease progression, number of CR sessions completed, and PC over preventing recurrence. However, participants in the current study were older (mean. $=63.84 ; t(207)=-2.67$, $p=.008$ ), endorsed fewer depressive symptoms (mean. $=$ 8.64; $t(195)=2.74, p=.007)$, and reported more PC over recovery (mean. $=3.12 ; t(206)=-2.94, p=.004)$ than those excluded from the study (means $=59.44,12.44$, and 2.82, respectively). Thus, caution is warranted when extrapolating these results to other CR populations.

\subsection{Measures}

The self-reported questionnaire packets included standardized measures and items adapted specifically for this study. In addition, demographic information was collected at Time 1 and medical information (including the number of completed CR sessions) was abstracted from the patients' medical records at the end of CR.

\subsubsection{Perceived control}

Two questions adapted from the Breast Cancer Perceived Control Scale ${ }^{[23]}$ were used at Time 1 to assess participants' PC over recovering from their current cardiac event ("In general, how much personal control do you think you have in recovering from your cardiac event?") and preventing a recurrent cardiac event ("How much personal control do you think you have in preventing another cardiac event?"). Response options were on a four-point scale ( 1 = very little, $4=$ total $)$, with higher scores indicating more PC.

\subsubsection{Depressive symptoms}

Time 1 depressive symptoms were measured using the 21item Beck Depression Inventory-II (BDI-II), ${ }^{[24]}$ which is a commonly-used measure for assessing depressive symptom severity. Items were on a four-point response scale ranging from 0 to 3 (e.g., 0 = I do not feel sad, $3=\mathrm{I}$ am so sad and unhappy that I can't stand it), with higher scores indicating more severe symptoms of depression. The scale has evidenced excellent reliability in both outpatient $(\alpha=.92)$ 
and college student $(\alpha=.93)$ samples. $^{[24]}$ The BDI-II had a reliability coefficient of .92 in this sample.

\subsubsection{Anxiety symptoms}

Anxiety symptoms were measured using the 21-item Beck Anxiety Inventory $(\mathrm{BAI})^{[25]}$ at Time 1 . The BAI is a commonly-used measure for assessing levels of anxiety. Response options ranged from 0 (not at all) to 3 (severely-it bothered me a lot), with higher summed scores indicating more severe symptoms of anxiety. The scale has shown excellent reliability $(\alpha=.92)$ with psychiatric outpatient populations ${ }^{[25,26]}$ and patients undergoing a CR program $(\alpha=$ .89). ${ }^{[27]}$ The BAI had a reliability coefficient of .90 in this sample.

\subsubsection{Adherence to health behavior recommendations}

Eight items were adapted from the Health Behaviors Scale ${ }^{[28]}$ to assess adherence to health behavior recommendations at Times 1 and 2. The scale asked questions about diet, exercise, and smoking behaviors over the last four weeks. Response options were on a seven-point scale $(1=$ never, $7=$ more than once per day) for diet and exercise items, and on a six-point scale ( $1=$ none, $6=$ more than 40 per day) for cigarette use. Previous research ${ }^{[29]}$ using a 7 -item version of this scale showed that it had moderately acceptable reliability $(\alpha=.64)$. In an attempt to improve reliability, the items were recoded to indicate whether the participant's behaviors met (1) or did not meet (0) health behavior recommendations based on existing guidelines and current literature. ${ }^{[30-32]}$ A composite score was then calculated by summing the responses to each item in order to determine overall adherence to the recommended cardiac-related health behaviors. Higher scores indicated greater levels of adherence. Reliabilities for the scale were KR20 $=.57$ at Time 1 and KR20 $=.64$ at Time 2 .

\subsection{Data analysis}

The statistical program SPSS $23.0^{[33]}$ was used to calculate descriptive and inferential statistics. The PROCESS macro add-on for SPSS ${ }^{[34]}$ was used to the test the proposed mediation hypotheses. These models examined PC as the predictor, symptoms of anxiety and depression as mediators, and health behavior adherence as the outcome (controlling for baseline levels). The PROCESS macro add-on utilizes an ordinary least squares (OLS) regression approach.

Preliminary data screening and descriptive statistics indicated that the data violated the assumption of normality required by OLS regression: The BDI-II variable was positively skewed and kurtotic. Conditional process analysis employs a bootstrapping procedure that is theoretically robust to violations of non-normality. However, in an effort to improve the distribution statistics, log transformations were applied to the
BDI-II variable. Skew and kurtosis improved significantly, so analyses were re-run using the log transformed BDI-II variable as the mediator. As expected, results remained largely the same as the original analyses. In addition, all other assumptions required by OLS regression were met. Therefore, to facilitate interpretation, only results from the original analyses were retained and will be presented here. In preliminary analyses, age was found to be significantly correlated with Time 1 BDI-II scores and Time 2 health behavior adherence. Therefore, age was entered as a covariate along with Time 1 health behavior adherence in each mediational analysis.

\section{RESULTS}

\subsection{Participant characteristics}

Table 1 displays the participant characteristics. The average age of study participants was 63.84 years $(\mathrm{SD}=9.98)$. The majority of participants was male and European American. Most participants completed at least some college and earned an annual income of $\$ 50,000$ or more. The most common cardiac diagnoses were stent placement and MI with stent placement. The CR staff assigned risk stratifications for disease progression based on the American Association of Cardiovascular and Pulmonary Rehabilitation guidelines. ${ }^{[35,36]}$ Almost all of the participants were stratified as low or medium risk for disease progression. Participants completed an average of 16.29 CR sessions ( $\mathrm{SD}=6.87$ ).

Table 2 shows descriptive statistics for each variable and correlations among variables. Participants, on average, reported perceiving moderate to a lot of control over their ability to prevent another cardiac event, and a lot of control over their ability to recover from their current cardiac event. The average level of psychological distress was relatively low, with $82 \%$ of the sample endorsing minimal levels of depression as defined by the BDI-II cut-off range of 0 to 13. ${ }^{[24]}$ The remaining participants endorsed mild (8\%) or moderate symptoms $(6 \%)$, with very few reporting severe depressive symptomatology (4\%). Anxiety symptoms were similar, with $70 \%$ of the sample reporting minimum symptoms as defined by the BAI range of 0 to $9 .{ }^{[25]}$ Far fewer participants endorsed mild (17\%), moderate (10\%), or severe (3\%) symptoms. Regarding adherence to health behaviors, participants reported moderate levels at both time points.

\subsection{Mediation analyses}

The first model included PC over prevention as the predictor (see Table 3), and the second included PC over recovery as the predictor (see Table 4). Results of Model 1 showed that the overall model was significant. However, bootstrapped confidence intervals for the indirect effects contained zero, meaning that depressive and anxiety symptoms did not signif- 
icantly mediate the relationship between PC over prevention and health behavior adherence. In fact, PC over prevention was unrelated to anxiety symptoms and neither anxiety nor depressive symptoms predicted adherence. However, PC over prevention was negatively related to depressive symptoms, and had a significant direct effect on Time 2 health behavior adherence, after controlling for covariates (baseline adherence and age). Thus, PC over prevention at the beginning of CR had a negative cross-sectional association with distress, but had a positive longitudinal effect on health behavior adherence at the end of CR.

Table 4 shows results from Model 2, which was also significant. Similar to Model 1, results showed that neither depressive nor anxiety symptoms significantly mediated the relationship between PC over recovery and adherence. Neither anxiety nor depressive symptoms were related to adherence. However, PC over recovery had significant direct effects on anxiety symptoms, depressive symptoms, and Time 2 health behavior adherence, after controlling for the covariates. Thus, PC over recovery at the beginning of CR had negative cross-sectional associations with distress and a positive longitudinal effect on health behavior adherence at the end of CR.

\section{Discussion AND CONCLUSION}

This study examined relationships between PC, symptoms of psychological distress, and adherence to health behavior recommendations among patients completing a CR program. We tested whether the relationship between baseline PC and health behavior adherence at the end of CR was mediated by symptoms of anxiety and depression. Results failed to support mediation, suggesting that PC does not operate through psychological distress to affect health behavior adherence over time. These findings were unexpected, as multiple studies have linked PC with symptoms of psychological distress $^{[11]}$ and, in turn, psychological distress with engagement in cardiac-related health behaviors. ${ }^{[3,7,9]}$ For example, a recent in-depth review ${ }^{[3]}$ points to a strong association between anxiety and depressive symptoms and health behavior adherence. In fact, health behaviors were identified as the primary mechanism through which distress impacted CVD outcomes.

Table 1. Participant characteristics

\begin{tabular}{|c|c|}
\hline Participant Characteristics & Number (\%) \\
\hline \multicolumn{2}{|l|}{ Gender } \\
\hline Male & $101(69.2)$ \\
\hline Female & $45(30.8)$ \\
\hline \multicolumn{2}{|l|}{ Race/Ethnicity } \\
\hline European American & 137 (94.5) \\
\hline African American & $5(3.4)$ \\
\hline Other & $3(2.1)$ \\
\hline \multicolumn{2}{|l|}{ Marital Status } \\
\hline Married or Stable Union & 107 (73.3) \\
\hline Other & 39 (26.7) \\
\hline \multicolumn{2}{|l|}{ Education } \\
\hline Less than High School & $4(2.8)$ \\
\hline High School/GED & $36(24.8)$ \\
\hline Some college/trade school & $30(20.7)$ \\
\hline 2- or 4-year college degree & $48(33.1)$ \\
\hline Graduate degree & 27 (18.6) \\
\hline \multicolumn{2}{|l|}{ Income } \\
\hline$<\$ 10,000$ & $6(4.1)$ \\
\hline$\$ 10,000-29,999$ & $24(16.4)$ \\
\hline$\$ 30,000-49,999$ & $23(15.7)$ \\
\hline$\$ 50,000-69,999$ & $22(15.1)$ \\
\hline Greater than $\$ 70,000$ & $62(42.4)$ \\
\hline Not reported & $9(6.3)$ \\
\hline \multicolumn{2}{|l|}{ Common Diagnoses } \\
\hline Stent & $47(32.2)$ \\
\hline MI \& Stent & $20(13.7)$ \\
\hline CABG & $31(21.2)$ \\
\hline Valve replacement/repair & $13(8.9)$ \\
\hline Other & $35(24.0)$ \\
\hline \multicolumn{2}{|l|}{ Risk stratification } \\
\hline Low & $70(47.9)$ \\
\hline Medium & 48 (32.9) \\
\hline High & $4(2.7)$ \\
\hline No risk stratification & $24(16.5)$ \\
\hline
\end{tabular}

Note. MI: Myocardial infarction; CABG: Coronary artery bypass graft

Table 2. Descriptive statistics and Pearson correlations for all study variables

\begin{tabular}{llllllll} 
& $\mathbf{1}$ & $\mathbf{2}$ & $\mathbf{3}$ & $\mathbf{4}$ & $\mathbf{5}$ & $\mathbf{6}$ & $\mathbf{7}$ \\
\hline 1. PC over Prevention & -- & $.61^{*}$ & -.09 & -.15 & $-.17^{*}$ & .07 & -.10 \\
2. PC over Recovery & & -- & $-.19 *$ & -.26 & .03 & $.22^{*}$ & $-.18^{*}$ \\
3. Anxiety Symptoms & & & -- & $.77^{* *}$ & -.22 & -.15 & -.25 \\
4. Depressive Symptoms & & & -- & $-.26^{* *}$ & $-.17^{*}$ & $-.25^{* *}$ \\
5. T1 Health Behaviors & & & & -- & $.59^{* *}$ & $.24^{* *}$ \\
6. T2 Health Behaviors & & & & & -- & $.18^{*}$ \\
7. Age & & & & & & -- \\
Mean. & 2.80 & 3.12 & 7.68 & 8.48 & 4.26 & 5.50 & 63.84 \\
SD & .75 & .62 & 7.84 & 7.86 & 1.76 & 1.56 & 9.98 \\
\hline
\end{tabular}

${ }^{*} p<.05,{ }^{* *} p<.01$; PC: Perceived control; T1: Time 1; T2: Time 2 
Table 3. Results of the mediation model for the relationship between prevention control, BAI and BDI-II, and adherence to health behaviors

\begin{tabular}{|c|c|c|c|c|c|c|c|c|c|c|c|c|}
\hline \multicolumn{13}{|l|}{ Consequent } \\
\hline \multirow{2}{*}{ Antecedent } & & \multicolumn{3}{|c|}{$M_{1}$ (BAI Score) } & & \multicolumn{3}{|c|}{$M_{2}$ (BDI-II Score) } & & \multicolumn{3}{|c|}{ Y (T2 Health Behaviors) } \\
\hline & & Coeff & $S E$ & $p$ & & Coeff & $S E$ & $p$ & & Coeff & $S E$ & $p$ \\
\hline X (Prevention PC) & $a_{1}$ & -1.50 & 0.84 & .076 & $a_{2}$ & -2.19 & 0.83 & .009 & $c^{\prime}$ & 0.37 & 0.15 & .011 \\
\hline $\mathrm{M}_{1}$ (BAI Score) & & --- & --- & --- & & --- & --- & --- & $b_{1}$ & -0.00 & 0.02 & .915 \\
\hline $\mathrm{M}_{2}$ (BDI-II Score) & & --- & --- & --- & & --- & --- & -- & $b_{2}$ & 0.00 & 0.02 & .745 \\
\hline \multirow[t]{3}{*}{ Constant } & $i_{\mathrm{M} 1}$ & 26.68 & 4.95 & .000 & $i_{\mathrm{M} 2}$ & 29.64 & 4.87 & .000 & $i_{\mathrm{Y}}$ & 1.60 & 0.94 & .090 \\
\hline & \multicolumn{4}{|c|}{$R^{2}=.112$} & \multicolumn{4}{|c|}{$R^{2}=.145$} & \multicolumn{4}{|c|}{$R^{2}=.378$} \\
\hline & \multicolumn{4}{|c|}{$F(3,142)=6.00, p<.001$} & \multicolumn{4}{|c|}{$F(3,142)=8.00, p<.001$} & \multicolumn{4}{|c|}{$F(5,140)=17.00, p<.001$} \\
\hline
\end{tabular}

Note. Model controlled for Time 1 age and health behavior adherence; PC: Perceived control; BDI-II: Beck Depression Inventory-II; BAI: Beck Anxiety Inventory; T2: Time 2

Table 4. Results of the model for the relationship between recovery control, BAI and BDI-II, and adherence to heath behaviors

\begin{tabular}{|c|c|c|c|c|c|c|c|c|c|c|c|c|}
\hline \multicolumn{13}{|l|}{ Consequent } \\
\hline \multirow{2}{*}{ Antecedent } & & \multicolumn{3}{|c|}{$M_{1}$ (BAI Score) } & & \multicolumn{3}{|c|}{$M_{2}$ (BDI-II Score) } & & \multicolumn{3}{|c|}{ Y (T2 Health Behaviors) } \\
\hline & & Coeff & $S E$ & $p$ & & Coeff & $S E$ & $p$ & & Coeff & $S E$ & $p$ \\
\hline X (Recovery PC) & $a_{1}$ & -2.92 & 1.00 & .004 & $a_{2}$ & -3.80 & 0.97 & .000 & $c^{\prime}$ & 0.59 & 0.18 & .001 \\
\hline $\mathrm{M}_{1}$ (BAI Score) & & --- & --- & --- & & --- & --- & --- & $b_{1}$ & -0.00 & 0.02 & .984 \\
\hline M & & --- & --- & --- & & --- & --- & --- & $b_{2}$ & 0.01 & 0.02 & .055 \\
\hline \multirow[t]{3}{*}{ Constant } & $i_{\mathrm{M} 1}$ & 32.70 & 5.50 & .000 & $i_{\mathrm{M} 2}$ & 36.70 & 5.36 & .000 & $i_{\mathrm{Y}}$ & 0.44 & 1.07 & .685 \\
\hline & \multicolumn{4}{|c|}{$R^{2}=.144$} & \multicolumn{4}{|c|}{$R^{2}=.190$} & \multicolumn{4}{|c|}{$R^{2}=.397$} \\
\hline & \multicolumn{4}{|c|}{$F(3,142)=7.97, p<.001$} & \multicolumn{4}{|c|}{$F(3,142)=11.07, p<.001$} & \multicolumn{4}{|c|}{$F(5,140)=18.42 p<.001$} \\
\hline
\end{tabular}

Note. Model controlled for Time 1 age and health behavior adherence; PC: Perceived control over recovery; BDI-II: Beck Depression Inventory-II; BAI: Beck Anxiety Inventory; T2: Time 2

Our findings may differ from other studies because of the relatively affluent and healthy nature of this sample, as evidenced by its high level of socioeconomic status (SES) and the number of patients who were qualified as low in risk stratification for cardiac disease progression. Being relatively healthy and possessing a high level of resources (including access to quality healthcare) likely allowed these participants to adhere to important health behaviors. By extension, these resources likely buffered against any additional aversive mental or physical health sequelae typically experienced following a cardiac event. Indeed, this sample evidenced relatively low levels of symptoms of depression and anxiety, which differs from research on patients with CVD in general. ${ }^{[3-6]}$ However, studies that specifically focus on patients in $\mathrm{CR}^{[20,37,38]}$ have also found levels of psychological distress to be in the minimal range as measured by the $\mathrm{BAI}^{[25]}$ and BDI-II. ${ }^{[24]}$ Therefore, these findings point to a potential distinction between larger groups of individuals with CVD and a sub-set of those who attend CR: patients in CR programs may experience fewer distress symptoms on account of their SES and other tangible resources. In fact, the very nature of CR programs (which require patients to have resources, such as

transportation and insurance) may inadvertently select out individuals classified as ethnic minorities or low in SES.

Although PC did not operate through symptoms of psychological distress to affect health behavioral adherence, PC was directly and concurrently (negatively) related to distress, as well as directly and prospectively (positively) related to adherence. Taken together, results suggest that PC may represent a psychosocial construct that can have proximal benefits to patients through brief intervention by CR staff. Participants in this sample reported levels of perceived control in the moderate to high range. These levels of PC are consistent with prior studies ${ }^{[10,17,18]}$ and suggest that individuals with CVD have similar levels of PC inside and outside CR. These levels of PC also suggest there is room for improvement, which may serve to improve short- and long-term physical and psychological outcomes.

Our finding that PC is concurrently and negatively related to experiencing distress symptoms is consistent with predictions from the reformulated learned helplessness model. ${ }^{[11]}$ It suggests that strategies aimed at enhancing PC may reduce distress, which could have a cascade of protective effects on health. For example, one investigation ${ }^{[39]}$ found that an 
increase in PC over the course of CR was predictive of lower levels of anxiety and depressive symptoms two months after CR completion. In addition, decreases in distress symptoms predicted better mental health outcomes six months later. Although we did not find evidence that distress symptoms predict health behavior adherence, abundant evidence ${ }^{[3,4]}$ suggests that distress is associated with poor morbidity and mortality in patients with CVD over time. Therefore, interventions to increase PC would undoubtedly have other psychological and physical health benefits.

Of most importance, our study documented longitudinal effects of both forms of PC on adherence to health behavior recommendations. This finding suggests that any increases in PC over cardiac event recovery and prevention may translate to more motivation and confidence to engage in behaviors that adhere to their health professionals' recommendations. Examples of adherence behaviors include completion of a CR program, taking cardioprotective medications as prescribed, and adopting heart-healthy diets. The present results thus have important implications for nurses caring for patients in CR, namely the need for developing targeted interventions aimed at enhancing levels of PC. Although research on well-tailored interventions is limited, some research has demonstrated the positive impact of psychosocial interventions on control appraisals. Doering and colleagues, ${ }^{[40]}$ for example, showed that a cognitive behavioral therapy (CBT) intervention for depression significantly increased levels of $\mathrm{PC}$ in patients who recently underwent a CABG or valve replacement/repair and were diagnosed with major or minor depression. The intervention ${ }^{[41]}$ consisted of eight in-home individual therapy sessions, with classic CBT techniques, including identifying and challenging negative automatic thoughts, problem-solving, learning coping strategies, and engaging in enjoyable behaviors. The authors argued that the reframing of negative thoughts likely helped participants to have a more balanced assessment of their level of control over their cardiac health. Prior research ${ }^{[42]}$ has also posited a link between behavioral activation and greater control appraisals. Of note, the intervention was delivered by advanced practice nurses, providing evidence for the feasibility of this type of intervention outside of behavioral health settings. Future research should assess the efficacy of adapted versions of these more intensive interventions. Given CBT's level of empirical support, it is likely that abridged versions would yield beneficial results within a CR setting.

Other researchers ${ }^{[10,17]}$ have also offered suggestions for enhancing PC in patients diagnosed with heart disease in a less formal format than CBT, including encouraging patients to be accountable for their illness through the modification of specific behaviors (e.g., diet, exercise); providing detailed information about the patient's disease and effective management strategies; providing patients with choices in CR programs and offering multiple options to cardiac care; engaging patients in developing their own plans of care with the guidance of medical providers; eliciting questions from patients to ensure they understand medical recommendations and guidelines, filling in gaps when needed; and encouraging patients to advocate for themselves. The benefits of enhancing self-efficacy have also been documented. ${ }^{[43]}$ Although self-efficacy is conceptually different from the broader forms of PC examined here, it is likely that fostering one's selfefficacy would generalize to greater control appraisals in other areas as well.

Despite the strengths of the study, there are limitations worth noting. First, the study relied solely on self-report measures which introduces the risk of mono-method and response biases. In addition, single-item measures were used to capture PC. Although researchers have effectively used similar items in the past, ${ }^{[23]}$ the use of single-item measures has been criticized because reliability coefficients cannot be calculated and multi-item measures are more likely to capture the full range of a psychological construct. ${ }^{[4]}$ Another psychometric shortcoming was the less than desirable reliability coefficients for the Health Behaviors Scale. ${ }^{[28]}$ The most likely explanation for this is due to the fact that the scale taps into a variety of behaviors. Although the scale is considered a unidimensional measure of health behaviors, it cannot be assumed that when someone engages in one health behavior (e.g., eating a healthy diet) that he/she will automatically adhere to others (e.g., exercising regularly). Thus, some of the items may not correlate at a high rate, thereby impacting the internal consistency of the scale. In addition, the analyzed relationships between PC and psychological distress were cross-sectional. Thus, it is difficult to confirm the directional nature of the relationship between these variables. Finally, the generalizability of the current findings to a wider CVD population may be limited by the characteristics of the participants, as the majority was European American, welleducated, and affluent. Guided by these limitations, future research should aim to use more targeted measures of health behaviors as opposed to a global score. Efforts should also be made to conduct longitudinal studies with more diverse patient populations.

In conclusion, findings suggest that levels of PC should be assessed upon entrance to CR. If patients endorse low levels, further assessment and intervention may be warranted to prevent aversive outcomes and facilitate recovery. In particular, these results suggest that low PC may be experienced concurrently with distress; because of the established relationship between distress and health outcomes in patients with CVD, 
targeting PC may also improve mental health. Furthermore, findings suggest that encouraging PC relevant to CVD recovery and prevention can have a positive effect on adherence to recommended health behavior changes within CR. Thus, any psychoeducational or clinical efforts to improve $\mathrm{PC}$ in

\section{REFERENCES}

[1] Benjamin EJ, Blaha MJ, Chiuve SE, et al. Heart Disease and stroke statistics-2017 Update: A report from the American Heart Association. Circulation. 2015 Mar 7; 135(10): 146-603.

[2] Heidenreich PA, Trogdon JG, Khavjou OA, et al. Forecasting the future of cardiovascular disease in the United States: A policy statement from the American Heart Association. Circulation. 2011; 123(8): 933-44. PMid:21262990 https://doi.org/10.1161/CIR.0b01 3e 31820 a55f5

[3] Cohen BE, Edmondson D, Kronish IM. State of the art review: Depression, stress, anxiety, and cardiovascular disease. Am J Hypertens. 2015; 28(11): 1295-302. PMid:25911639 https://doi .org/10.1 093/ajh/hpv047

[4] Watkins LL, Koch GG, Sherwood A, et al. Association of anxiety and depression with all-cause mortality in individuals with coronary heart disease. J Am Heart Assoc. 2013; 2(2): 1-11.

[5] Lichtman JH, Bigger JT, Blumenthal JA, et al. Depression and coronary heart disease: Recommendations for screening, referral, and treatment: A science advisory from the American Heart Association Prevention Committee of the Council on Cardiovascular Nursing, Council on Clinical Cardiology, Council on Epidemiology and Prevention, and Interdisciplinary Council on Quality of Care and Outcomes Research: Endorsed by the American Psychiatric Association. Circulation. 2008; 118(17): 1768-75. PMid:18824640 https://doi.org/10.1161/CIRCULATIONAHA.108.190769

[6] Grace SL, Abbey SE, Irvine J, et al. Prospective examination of anxiety persistence and its relationship to cardiac symptoms and recurrent cardiac events. Psychother Psychosom. 2004; 73(6): 344-52. PMid:15479989 https://doi .org/10.1159/000080387

[7] Duivis HE, de Jonge P, Penninx BW, et al. Depressive symptoms, health behaviors, and subsequent inflammation in patients with coronary heart Disease: Prospective findings from the Heart and Soul Study. Am J Psychiatry. 2011 Sep; 168(9): 913-20.

[8] Davies SJC, Allgulander C. Anxiety and cardiovascular disease. In: Anxiety Disorders. 2013. 85-97.

[9] Whooley MA, de Jonge P, Vittinghoff E, et al. Depressive symptoms, health behaviors, and risk of cardiovascular events in patients with coronary heart disease. JAMA. 2008 Nov 26; 300(20): 2379-88.

[10] Moser D, Dracup K. Psychosocial recovery from a cardiac event: the influence of perceived control. Hear Lung J Acute Crit Care. 1995; 24(4): 273-80. https://doi.org/10.1016/S0147-9563(05)8 0070-6

[11] Abramson LY, Seligman ME, Teasdale JD. Learned helplessness in humans: Critique and reformulation. J Abnorm Psychol. 1978; 87(1): 49-74. https://doi.org/10.1037/0021-843X.87.1.49

[12] Brown JD, Siegel JM. Attributions for negative life events and depression: The role of perceived control. J Pers Soc Psychol. 1988; 54(2): 316-22. https://doi.org/10.1037/0022-3514.54.2.316

Published by Sciedu Press patient populations may have a cascade of positive effects on proximal and distal physical and psychological health outcomes.

\section{CONFlicts OF INTEREST Disclosure}

The authors declare that there is no conflict of interest.
[13] Chorpita BF, Barlow DH. The development of anxiety disorders: The role of control in the early environment. Psychol Bull. 1998; 124(1): 3-21. https://doi.org/10.1037/0033-2909.124.1.3

[14] Sweeney PD, Anderson K, Bailey S. Attributional style in depression: A meta-analytic review. J Pers Soc Psychol. 1986; 50(5): 974-91. https://doi.org/10.1037/0022-3514.50.5.974

[15] Dweck CS, Wortman CB. Learned helplessness, anxiety, and achievement motivation: Neglected parallels in cognitive, affective, and coping responses. Ser Clin Community Psychol Achiev Stress Anxiety. 1982; 93-125.

[16] Banerjee T, Kyoung SL, Browning SR, et al. Limited association between perceived control and health-related quality of life in patients with heart failure. J Cardiovasc Nurs. 2014; 29(3): 227-31.

[17] Dracup K, Westlake C, Erickson VS, et al. Perceived control reduces emotional stress in patients with heart failure. J Hear Lung Transplant. 2003; 22(1): 90-3. https://doi.org/10.1016/S1053-2 498(02) 00454-0

[18] Gallagher R, McKinley S. Anxiety, depression and perceived control in patients having coronary artery bypass grafts. J Adv Nurs. 2009 Nov; 65(11): 2386-96.

[19] Heo S, Lennie TA, Pressler SJ, et al. Factors associated with perceived control and the relationship to quality of life in patients with heart failure. Eur J Cardiovasc Nurs. 2015 Apr 15; 14(2): 137-44.

[20] Kidd T, Poole L, Leigh E, et al. Health-related personal control predicts depression symptoms and quality of life but not health behaviour following coronary artery bypass graft surgery. J Behav Med. 2016 Feb 4; 39(1): 120-7.

[21] Barry LC, Kasl SV, Lichtman J, et al. Perceived control and change in physical functioning after coronary artery bypass grafting: A prospective study. Int J Behav Med. 2006 Sep; 13(3): 229-36.

[22] Fritz MS, MacKinnon DP. Required Sample Size to Detect the Mediated Effect. Psychol Sci. 2007 Mar 4; 18(3): 233-9.

[23] Bennett KK, Compas BE, Beckjord E, et al. Self-blame and distress among women with newly diagnosed breast cancer. J Behav Med. 2005; 28(4): 313-23. PMid:16049635 https://doi.org/10.100 7/s10865-005-9000-0

[24] Beck A, Steer R, Brown G. Beck Depression Inventory-II. San Antonio: Pearson; 1996.

[25] Beck AT, Epstein N, Brown G, et al. An inventory for measuring clinical anxiety: Psychometric properties. J Consult Clin Psychol. 1988; 56(6): 893-7. https://doi.org/10.1037/0022-006X.56 .6 .893

[26] Fydrich T, Dowdall D, Chambless DL. Reliability and validity of the beck anxiety inventory. J Anxiety Disord. 1992 Jan; 6(1): 55-61.

[27] Clark JMR, Marszalek JM, Bennett KK, et al. Comparison of factor structure models for the Beck Anxiety Inventory among cardiac rehabilitation patients. J Psychosom Res. 2016 Oct; 89: 91-7.

[28] Naslund GK, Fredrikson M. Health behavior, knowledge and attitudes among Swedish university students. Scand J Psychol. 1993 Sep; 34(3): 197-211. 
[29] Bennett KK, Marte RM. Patient attributions for cardiac events: predictors of physical and psychological recovery. J Appl Soc Psychol. 2013 Mar; 43(3): 660-72.

[30] Appel LJ, Frohlich ED, Hall JE, et al. The importance of populationwide sodium reduction as a means to prevent cardiovascular disease and stroke: A call to action from the American Heart Association. Circulation. 2011 Mar 15; 123(10): 1138-43.

[31] Haskell WL, Lee IM, Pate RR, et al. Physical activity and public health: Updated recommendation for adults from the American College of Sports Medicine and the American Heart Association. Med Sci Sports Exerc. 2007 Aug; 39(8): 1423-34.

[32] Lichtenstein AH, Appel LJ, Brands M, et al. Diet and lifestyle recommendations revision 2006: A scientific statement rrom the American Heart Association Nutrition Committee. Circulation. 2006 Jun 26; 114(1): 82-96.

[33] IBM Corp. IBM SPSS Statistics for Windows. Version 23.0 [software]. 2015.

[34] Hayes AF. Introduction to mediation, moderation, and conditional process analysis: A regression-based approach. Guilford Press: New York; 2013.

[35] Balady GJ, Williams MA, Ades PA, et al. Core components of cardiac rehabilitation/secondary prevention programs: 2007 update. J Cardiopulm Rehabil Prev. 2007 May; 27(3): 121-9.

[36] Smith SC, Benjamin EJ, Bonow RO, et al. AHA/ACCF Secondary prevention and risk reduction therapy for patients with coronary and other atherosclerotic vascular disease: 2011 update: A Guideline from the American Heart Association and American College of Cardiology Foundation. Circulation. 2011 Nov 29; 124(22): 2458-73.

[37] Kreikebaum S, Guarneri E, Talavera G, et al. Evaluation of a holistic cardiac rehabilitation in the reduction of biopsychosocial risk factors among patients with coronary heart disease. Psychol Health Med.
2011; 16(3): 276-90. PMid:21491336 https ://doi.org/10.108 $0 / 13548506.2010 .542170$

[38] McGrady A, Burkes R, Badenhop D, et al. Effects of a brief intervention on retention of patients in a cardiac rehabilitation program. Appl Psychophysiol Biofeedback. 2014; 39: 163-70. PMid:25150038 https://doi.org/10.1007/s10484-014-9252-y

[39] Michie S, O'Connor D, Bath J, et al. Cardiac rehabilitation: The psychological changes that predict health outcome and healthy behaviour. Psychol Heal Med. 2005; 10(1): 88-95. https://doi.or $\mathrm{g} / 10.1080 / 13548500512331315398$

[40] Doering LV, McGuire A, Eastwood JA, et al. Cognitive behavioral therapy for depression improves pain and perceived control in cardiac surgery patients. Eur J Cardiovasc Nurs. 2016; 15(6): 417-24. PMid:26115954 https://doi.org/10.1177/14745151155922 92

[41] Doering LV, Chen B, Cross Bodán R, et al. Early Cognitive Behavioral Therapy for Depression After Cardiac Surgery. J Cardiovasc Nurs. 2013; 28(4): 370-9. PMid:22635060 https://doi.org/10 .1097/JCN.0b013e31824d967d

[42] Mcevoy P, Law A, Bates R, et al. Using behavioural activation in the treatment of depression: A control theory perspective. J Psychiatr Ment Health Nurs. 2013; 20(10): 890-5. PMid:23240823 https://doi.org/10.1111/jpm. 12032

[43] Marks R, Allegrante JP. A review and synthesis of research evidence for self-efficacy-enhancing interventions for reducing chronic disability: Implications for health education practice (Part II). Health Promot Pract. 2005 Apr 30; 6(2): 148-56.

[44] Hoeppner BB, Kelly JF, Urbanoski KA, et al. Comparative utility of a single-item versus multiple-item measure of self-efficacy in predicting relapse among young adults. J Subst Abuse Treat. 2011 Oct; 41(3): 305-12. 